\title{
MJIT - Manutenção por Just in Time Manutenção Otimizante Realizada no Tempo Exato Baseado em Condições Operacionais
}

\author{
Michitoshi Oishi - Prof. Dr.
}

Universidade Estadual de Ponta Grossa

Rua Dr. Alexandre Gutierrez, 863 - Apt. 91. CEP 80240-130

\section{Carlos Augusto Candêo Fontanini}

\section{Mestrando em Administração-Universidade Federal do Paraná}

Palavras-chave: Sistema de manufatura, manutenção, redução nas paradas de produção, produtividade, just in time.

Key words: manufacture system, maintenance, decreasing of production pauses, productivity, just in time.

\section{RESUMO}

O presente artigo investigou como as fábricas japonesas desenvolvem sua manutenção para redução das paradas de produção. Isto aumenta a qualidade e produtividade, evitando atraso na entrega dos produtos e satisfazendo as necessidades dos clientes.

Além do TPM-Total Productive Maintenance, existe outro sistema de manutenção baseado nas condições operacionais do equipamento, realizando a manutenção no momento necessário, que pode ser chamado de MJIT. Manutençăo Por Jus In Time.

Este sistema evita a manutenção baseada na estimativa de vida útil do equipamento que pode realizar a manutenção antes do momento necessário, como depois da quebra.

Uma pesquisa qualitativa realizada em fábricas brasileiras, identificou o alto potencial e necessidade de implantação de um sistema de manutenção similar ao MJIT.

\begin{abstract}
This article investigated how japanese factorys develop their maintenance to reduce productions pauses. It increases productivity and quality, avoides products late delivery and satisfies customers needs.

Besides TPM-Total Productive Maintenance, there is another maintenance system based on equipment operational conditions doing the maintenance in the exact moment of necessity that can be called MJITMaintenance by Just In Time. It avoids unnecessary maintenance based on the estimated life time that may conduct to realize maintenance before the real necessity or after broken.

A qualitative research in brasilian factorys identified a high potential and need of a mainenance system similar to $M J I T$.
\end{abstract}

Belo Horizonte, Vol 7, $\mathrm{N}^{\circ} 1$, p.75-83

Jul.1997 


\section{PRODUÇÃO}

\section{Introdução}

$O$ presente trabalho apresenta o que ocorre no desenvolvimento da tecnologia de manutenção no Japão e sua estrutura, minimizando as falhas humanas nas percepções sobre os sintomas que os equipamentos apresentam em operação, estimativa de vida útil ou previsão das possíveis paradas acidentais.

Para tal objetivo, apresentaremos no início o conceito da necessidade de desenvolvimento de equipamentos e a manutenção de melhor desempenho na operação, seguida da descrição da importância da participação humana e suas limitações enfatizando a necessidade da tecnologia de automação onde as sensibilidades, conhecimentos ou experiências não seriam capazes de prever as possíveis falhas que ocorrem ou podem ocorrer nos instantes futuros.

Também apresentaremos os resultados da pesquisa realizada junto a algumas empresas do Japão, e no final deste, a existência de grande potencialidade de implementação em indústrias brasileiras de um sistema similar ao MJITManutenção Por Just in Time, que consiste na realização da manutenção no momento ideal, conduzindo ao aumento da produtividade, qualidade, evitando atrasos na entrega dos produtos, permitindo obter a melhoria em escala revolucionária no resultado operacional, que é de extrema importância neste momento de mudanças, como resultado da aceleração da globalização da economia e a competitividade desenvolvida em âmbito internacional.

\section{Melhoria dos Desempenhos Operacionais de Equipamentos e Aumento da Produtividade com a Inovação Tecnolócia de Manutenção}

Com toda a transformação e evolução que hoje estamos vivendo, é imprescindivel que nos adaptemos e nos antecipemos às mudanças, acompanhando todo esse desenvolvimento tecnológico, fazendo com que permaneçam no mercado apenas as indústrias que souberem administrar seus recursos, formando uma estrutura empresarial apta a sobreviver aos novos tempos.

A modernização e/ou melhoria da tecnologia de produção é um dos fatores fundamentais para o alcance deste objetivo.

Esta tecnologia de produção pode ser compreendida em três grupos e duas fases principais, conforme a seguir:

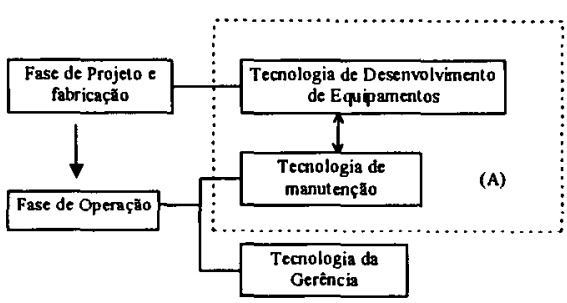




\section{PRODUÇÃO}

A tecnologia de desenvolvimento de equipamentos e a tecnologia de manutenção, é a tecnologia que trará inovação ao sistema de produção, principalmente; dando também a devida importância a tecnologia de gerência, para que juntos se obtenha a elevação brusca da qualidade e produtividade na empresa.

Sem o desenvolvimento do hardware (A), não será possível implantar um programa de manutenção eficaz, não permitindo desperdícios provocados por esses equipamentos, as paradas inesperadas e fabricação de produtos defeituosos.

A tecnologia de desenvolvimento de equipamentos e a tecnologia de manutenção, é desenvolvida na fase de projeto e fabricação, entretanto, a melhoria da tecnologia de manutenção pode ser implementada também na fase de operação, a qual poderá contribuir no melhor desempenho; que é justamente o objetivo deste trabalho.

A seguir descreveremos o que foi observado na pesquisa realizada no Japão sobre o MJIT.

\section{Os Resultados da Pesquisa no Japão e as Características do MJIT}

\section{1- Sistema de Controle Geral de Equipamentos de Produção}

Intensifica-se, hoje, a exigência da tecnologia de controle de equipamentos para estabilidade da produção e melhoria da qualidade de produtos, com o avanço tecnológico, desenvolvimento de processos contínuos e automação em diversas áreas no processo de produção.

Dentro desta concepção, existe MJIT - Manutenção por Just in Time que conduz o custo de manutenção ao mínimo.

Como meio para concretizaçã́o do MJIT, torna-se necessário Sistema Total estruturado, por Sistema Expert e Sistema de Diagnose, através do Monitoramento Remoto "On line".

Abordagem progressiva para concretização do Sistema Total com a aplicação eficiente de computadores e a tecnologia de sistematização é o problema cardial da estratégia de empresas na atualidade.

\section{Características do MJIT}

1. Concretização do Sistema Total abrangendo o Sistema de Gerenciamento (Controle de Produção, Controle de Manutenção).

2. Concretização do Sistema de Controle de Equipamentos "On line" interligando-se em rede em larga extensão.

3. Concretização do Sistema de Controle de Equipamentos interligado com o Sistema de Diagnose "On line".

4. Concretização do serviço adequado às necessidades diversas e reais, desde relativos a sensores isolados até ao Sistema Total. 


\subsection{Sistema de Monitoramento Remoto}

É destinado para monitoração no Tempo Real, os equipamentos e instalações em extensa área interligadas por rede (Ethernet, ME-NET, etc.) do sistema de diagose, computadores de processo, equipamentos mecatrônicos, etc.

\section{Características}

1. Monitoração de condições operacionais isoladas e em conjunto interligando por meio de rede, equipamentos de processo mecatrônicos de diferentes tipos, marcas e fornecedores.

2. É possivel aumento, indicação, alteração e redução de carga de máquinas por meio da programação no circuito.

3. Preparação dos gráficos, curvas de tempo, imagens de equipamentos sem programação.

3.3- Sistema de Controle de Equipamentos (Toda Fabrica)

Este sistema consiste de sete subsistemas que são: Sistema Padrão de Equipamentos, Sistema de Planejamento de Manutenção, Sistema de Verificação e Diagnose, Sistema de Controle de Execução de Reparos, Sistema de Inventários e Controle de Materiais, Sistema de Controle de Orçamento, e Sistema de Avaliação de Análise.
Este sistema controla o trabalho de reparos, estoques de sobressalentes, planejamento de orçamento, performance, coleta de dados e avaliação baseada em informaç̃̃es recebidas de diagnose de máquinas.

É possível também, serem interligados funcionalmente com o Sistema de Controle Operacional de toda fábrica e capazes de ajustar o plano para condição operacional.

\section{Caracteristicas}

1. Concretização do Sistema Total

1) Este sistema cobre todas as atividades de controle de equipamentos. (Padronização, Planejamento, Performance, Resultados e Avaliação)

2) É adaptado para todos os campos de controle de equipamentos. (Máquinas, Eletricidade, Química, Execução de obra).

2. Novo Sistema Padrão de Manutenção. Mudança da MBT Manutenção Baseada em Tempo para MJIT-Manutenção por Just in Time.(Expansão do Controle Quantitativo de Dados de Inspeção e Previsão de Vida Útil).

3. Unificação de Sistemas de Informações (Aperfeiçoamento Efetivo de Base de Dados) (Ação eficiente através de fácil verificação e análise).

4. Promoção da Sistematização de Controle Total de Equipamentos em "On line" por meio de conjunção dos Sistemas 
de Diagnose e de Monitoramento Remoto de Equipamentos.

5. Elevação da Eficiência das Atividades de Processamento de Documentos. Promoção da simplificação das atividades de processamento de desenhos e textos e automação de escritórios.

3.4- Sistema de Diagnose de Equipamentos (Toda Fabrica)

Este sistema realiza o monitoramento permanente das condições operacionais de equipamentos que influem nas operações e qualidade de produtos. Se acontecer qualquer anormalidade, realiza automaticamente, as investigações das causas através de matriz de causas e efeitos relativos a equipamentos e qualidade de produtos, e fornece as orientações necessárias para os operadores.

As informações e dados não considerados anormais são transferidos para o Sistema de Controle da Fábrica através da rede após processados para o uso para o controle de toda fábrica a médio e longo prazo.

Metodologias Aplicadas de Diagnose para Equipamentos e seus Conteúdos

\begin{tabular}{|c|c|c|}
\hline Método & Equipamentos & Conteúdo de Diagnose \\
\hline $\begin{array}{l}\text { 1. Diagnose de } \\
\text { Vibração }\end{array}$ & $\begin{array}{l}\text { Bombas, Motores, } \\
\text { Sopradores, Rolos }\end{array}$ & $\begin{array}{l}\text { Mancais (rolamentos, anéis } \\
\text { internos e externos, má } \\
\text { lubrificação), Entortamento de } \\
\text { eixos, Desgastes por desvio, Mau } \\
\text { alinhamento, Engrenagens (falta de } \\
\text { dentes, riscos), Base (falta de } \\
\text { resistência, desvios), Cavitação. }\end{array}$ \\
\hline $\begin{array}{l}\text { 2. Diagnose de } \\
\text { Mudança de } \\
\text { Posicionamento } \\
\end{array}$ & Turbinas, Bombas & $\begin{array}{l}\text { Entortamento de eixos, Desgaste } \\
\text { por desvio, Base (falta de } \\
\text { resistência), Desvios, Ressonância. }\end{array}$ \\
\hline $\begin{array}{l}\text { 3. Diagnose da } \\
\text { Temperatura }\end{array}$ & $\begin{array}{l}\text { Bombas, Motores, } \\
\text { Equipamentos de } \\
\text { Refrigeração }\end{array}$ & $\begin{array}{l}\text { Má lubrificação de eixos, Mal } \\
\text { enrolamento de fios, Sobrecarga, } \\
\text { Falta de meio refrigerante. }\end{array}$ \\
\hline $\begin{array}{l}\text { 4. Diagnose de } \\
\text { Acústica }\end{array}$ & Motores, Sopradores & $\begin{array}{l}\text { Barulho anormal de atrito, Falha da } \\
\text { hélice, Desbalanceamento. }\end{array}$ \\
\hline 5. Diagnose de $\mathrm{AE}$ & $\begin{array}{l}\text { Bombas, Tubulação, } \\
\text { Válvula }\end{array}$ & \begin{tabular}{ll|} 
Cavitação, & Vazamento, \\
Entupimento. & \\
\end{tabular} \\
\hline $\begin{array}{l}\text { 6. Diagnose de } \\
\text { Torque }\end{array}$ & Motores, Rolos, Redutores & Eixos, Pinos de trava. \\
\hline $\begin{array}{l}\text { 7. Diagnose de } \\
\text { Correntes } \\
\text { Elétricas } \\
\end{array}$ & \begin{tabular}{|l|} 
Motores, Limpadores de \\
encrustação, Correias \\
Transportadoras \\
\end{tabular} & $\begin{array}{l}\text { Penetração de sujeiras, Cargas } \\
\text { anormais, Correntes de transmissão } \\
\text { danificadas. }\end{array}$ \\
\hline $\begin{array}{l}\text { 8. Diagnose de } \\
\text { Pressão }\end{array}$ & $\begin{array}{l}\text { Bomba, Tubulação, } \\
\text { Tubulação de graxas, } \\
\text { Equipamentos de } \\
\text { refrigeracão }\end{array}$ & $\begin{array}{l}\text { Vazamento da tubulação, Falhas } \\
\text { nas válvulas }\end{array}$ \\
\hline
\end{tabular}




\section{PRODUÇÃO}

\section{Características}

1. Possui funções de diagnose para operações e qualidade.

2. Possui funções automáticas de diagnose.

3. Prepara automaticamente os relatórios.

4. Pode ser conectado com Sistema de Controle Operacional mais abrangente ou Sistema de Controle da Fábrica.

5. Possui funções de controle de dados utilizando disquetes de microcomputador, funções de Inteligência Artificial.

\section{5- Sistema de Processamento de Imagens}

(1) Sistema de Diagnose de Equipamentos por meio de Processamento de Imagens.

Este sistema identifica desajustes ou más condições operacionais quantitativamente através de informações de monitoramento feito por câmera de vídeo, acionando a sirene se 'necessário.

(2) Sistema de Base de Dados para Imagens de Equipamentos. Este sistema capta as imagens de equipamentos através de fotografias, memória fotográfica, câmera de video e apresenta-as no monitor ou imprimindo-as na impressora dependendo das necessidades.

\section{Características}

1. Este sistema é capaz de diagnosticar objetos em movimento.

2. Em função da necessidade, o operador poderá verificar as condições operacionais através do televisor-monitor.

3. Este sistema é capaz de combinar com o sistema de alarme de som.

4. É possível transmitir as imagens por fibras óticas.

\section{6- Sistema Expert}

Este sistema realiza julgamento sinteticamente, de toda fábrica, sobre a perfeição dos equipamentos, operação e a qualidade de produtos, utilizando respectivamente os sinais colocados nos equipamentos, indicadores de operação da fábrica, e das medidas de qualidade. Uma vez completada a medição dos sinais de processo e coletado seus dados, este sistema construirá, sistemas mais avançados utilizando os conhecimentos $e$ Know-how do pessoal de operação, de controle de qualidade e de manutenção.

\section{7- Sistema de A rquivos Eletrônicos}

Este sistema acessa ou registra as informações dentro de arquivos eletrônicos através da estação de trabalho ou micro computador ligado por LAN. 


\section{PRODUÇÃO}

\section{Caracteristicas}

1. Este sistema se conecta com o sistema aberto Arquitetura Aberta.

2. Este sistema utiliza processamento de alta velocidade na rede Processamento de dados de alta velocidade.

- Este sistema constrói o sistema de arquivos utilizando microcomputadores com núcleo.

- Pode ser conectado às variadas funções de comunicação. Suas funções podem ser ampliadas com a construção da rede; fácil utilização, é apresentado no Windows.

\section{MJIT nas Empresas Brasileiras}

Com o propósito de estabelecimento de análise comparativa do que foi encontrado em indústrias japonesas e brasileiras, foi feito um levantamento da situação das indústrias no Brasil, limitando-se em contatos verbais com os profissionais de projetos de sistemas de automação e/ou da manutenção de algumas indústrias de produção de processo contínuo na região sul, principalmente nos estados do Paraná e Santa Catarina, e constatou-se qualitativamente o seguinte:

a) Manutenção preventiva hoje realizada, baseia-se mais no tipo de uso e/ou estimativa de vidas úteis de equipamentos e/ou de seus componentes.

b) Existe alguns sistemas mais direcionados para controles de operação mas quase nada para manutenção preventiva baseada em condições operacionais. Existem somente para os equipamentos de modo isolado, por meio de captação de vibrações, temperaturas, pressão, etc.

c) Em vista da probabilidade da sua estimativa não coincidir com a realidade, tem acontecido as paradas antes da previsão, que causa prejuízos de vulto em diversos aspectos: aumento do custo na aquisição de componentes, reparos necessários fora da época prevista que juntamente com a perturbação das atividades normais e rotinas, parada de produção que gera a queda da receita, que se converte em prejuizo de valor quase equivalente, além do atraso do atendimento da entrega prevista para os clientes.

d) Ambiente desmotivante, justificado de insucessos em vez da busca da melhoria do desempenho global.

e) Ciclo negativo estabelecido que redunda na necessidade de sua quebra por procedimento novo, inovador, que não depende somente da sensibilidade, motivação, conhecimento, e experiências profissionais.

f) Potencial aumento da produtividade em empresas brasileiras com implantação MJIT. 
As pesquisas quantitativas serão realizadas e possíveis de serem apresentadas oportunamente.

\section{Potencialidade de Melhoria da Produtividade com MJIT nas Empresas Brasileiras}

Analisando as situações em que se encontram as empresas brasileiras, podemos constatar que existe grande potencialidade de obtenção de beneficios, com a implantação do MJT, conforme a seguir:

a) Possibilidade de planejamento, através das informações obtidas pela diagnose de todo sistema, planejamento este composto de manutenção e produção, ajustado nas condições reais das necessidades observadas, agregado com o planejamento de entrega dos pedidos.

b) Redução significativa de mão-deobra e materiais de manutenção aplicada em todo o processo produtivo, possibilitando prever as épocas de manutenção a efetuar.

c) Redução de erros na identificação das falhas pelo ser humano através da substituição por sistemas.

d) Como consequência dos itens descritos acima, obtemos o aumento do desempenho operacional e conseqüente aumento revolucionário da competitividade e a lucratividade.

\section{Conclusão}

Neste trabalho apresentamos a pesquisa realizada no Japão sobre a manutenção e observamos o resultado surpreendente com a aplicação do MJIT Manutenção por Just in Time, obtendo-se o aumento revolucionário de produtividade e conseqüente redução de custos.

Enquanto que, a pesquisa nas empresas brasileiras tem mostrado a existência de alguns esforços para monitoramento e diagnose de forma ainda bastante tímida com baixo resultado.

Assim sendo, vimos a grande potencialidade para aplicação do mesmo sistema, para obtenção de benefícios extraordinários que se pode esperar com sua introdução e aplicação, apesar que a pesquisa nas empresas brasileiras, limitou-se no mérito qualitativo.

Entretanto, a pesquisa pretende estender na aferição quantitativa, o que esperamos que chegue a mesma conclusão aqui obtida de forma qualitativa.

\section{Bibliografia}

HAYASHI, K. Busquemos o CIM original, Revista - Controles Industriais, Tokyo, 1992.

HIPKIN, I. B.; LOCKETT, A.G. A study of maintenance technology 
implementation. Omega, The International Journal of Management Science, vol. 23, n.1, p.79-88,1995.

JOSTES, R.; HELMS, M. M. Total productive maintenance and its link to total quality management. Work Study, vol.43, n.7, p.18-20,1994.

KANNO, E. Tudo sobre o CIM, Publicações da Universidade Sanno, Tokyo, 1993.

KAWASAKI TECHNOLOGICAL DEVELOPMENT DIVISION, Kawasaki Total Operation Control System, Tokyo, 1993.

MAGGARD, B. N.; RHYNE D. M. Total productive maintenance: a timely integration of production and maintenance. Production and Inventary Management Journal, vol. 33, n.4, p. 610, fourth quarter, 1992.

OISHI, M. TIPS - Técnicas integradas na produção e serviços. São Paulo: Pioneira,1995.

TAKAHASHI, Y., OSADA, T. TPMTotal productive maintenance. Asian Productivity Organization, Tokyo, 1990.

UMEDA, T. CIM e administração estratégica. Associação de Pesquisas Industriais, 1992, Tokyo. 